\title{
UEMS: focus sur la formation médicale postgraduée et continue ainsi que sur l'assurance-qualité
}

\section{Max Giger}

Past-président de l'Institut suisse pour la formation médicale postgraduée et continue ISFM
Correspondance:

Dr Max Giger Rosenrain 9

CH-8400 Winterthour

giger.max@bluewin.ch
Cette année, l’Union européenne des médecins spécialistes (UEMS) a invité ses membres à sa $52^{\mathrm{e}}$ assemblée générale organisée à Prague du 7 au 9 octobre 2010 par la société médicale tchèque. Pour la première fois, les délégués des sociétés nationales de médecine (council) ont siégé avec ceux des sociétés de discipline scientifique (sections and boards). Parmi les points les plus importants de l'ordre du jour, les délégués ont adopté la révision statutaire en chantier depuis cinq ans (laquelle tient compte du resserrement de la collaboration entre les organisations nationales de médecine et les sociétés de discipline médicale) et l'acquisition d'une «domus medica» dans le quartier de l'UE à Bruxelles.

Ces décisions renforcent la position, déjà reconnue en Europe, de l'UEMS dans son rôle précurseur pour la formation médicale postgraduée et continue et la qualité des soins. Les délégués ont accepté la création de trois comités, un comité permanent (standing committee) pour la formation postgraduée, un candidats est également évalué de manière décentralisée. L'ECAMSQ aspire à atteindre un standard élevé reconnu par l'Europe pour les compétences des spécialistes, à l'inverse de la tendance politique actuelle à tout niveler vers le bas. Les premiers résultats des trois sociétés pilotes (radiologie, cardiologie, anesthésiologie) sont attendus pour l'été 2011. Les diplômes de spécialiste sont remis selon le droit national. Les pays membres sont invités à coopérer. Les pays scandinaves saluent l'évaluation formative des compétences médicales mais ils rejettent l'évaluation sommative. Le CESMA poursuivra son travail mené jusqu'à présent sur les 27 évaluations européennes des spécialistes qui existent déjà (european boards). Notons que la réussite des european boards fait, entre autres, partie intégrante des examens suisses de spécialiste en ophtalmologie, anesthésiologie, chirurgie plastique, reconstructive et esthétique et en urologie.

Le comité pour la formation continue réunit l'instance d'accréditation pour la formation continue mé-

\section{Les décisions renforcent la position de l'UEMS dans son rôle précurseur pour la formation médicale postgraduée et continue et la qualité des soins}

pour la formation continue et un troisième pour la gestion de la qualité.

Le CESMA (Council for european speciality medical assessments), l'actuel groupe de travail sur la formation postgraduée, et la future plate-forme internet de la formation postgraduée pour l'accréditation des connaissances, des aptitudes et du professionnalisme (European council for the accreditation of medical specialist qualification/ECAMSQ) constituent le comité de la formation postgraduée. Ce regroupement permet de conjuguer les efforts en faveur de l'accréditation européenne de la formation postgraduée médicale et des examens de spécialiste. Les connaissances sont évaluées sur la base de questionnaires à choix multiples. Les aptitudes sont jugées de manière formative - comme en Grande-Bretagne, aux Pays-Bas et en Suisse - au moyen d'évaluations dans le milieu de travail [1]. Le professionnalisme des candidates et dicale qui fonctionne à merveille (european accreditation council for continuing medical education/ EACCME) et le groupe de travail constitué jusqu'à présent. Ce comité poursuit ses travaux sur la base de la Déclaration en faveur de la formation continue médicale des spécialistes européens de 1994 (charter on CME) et sur la base de la Déclaration de Bâle (2001) en vue d'un développement professionnel continu (continuous professional development/CPD).

Des soins médicaux de qualité élevée et conformes aux besoins des patients constituent l'objectif déclaré du comité pour la gestion de la qualité. Siègent dans ce comité l'european advisory council for quality management of specialist medical practice (EACQM) et le groupe de travail actuel.

La durée de mandat dans tous les organes de l'UEMS est désormais réglée de manière uniforme: elle est de quatre ans et ne peut être prolongée qu'une 


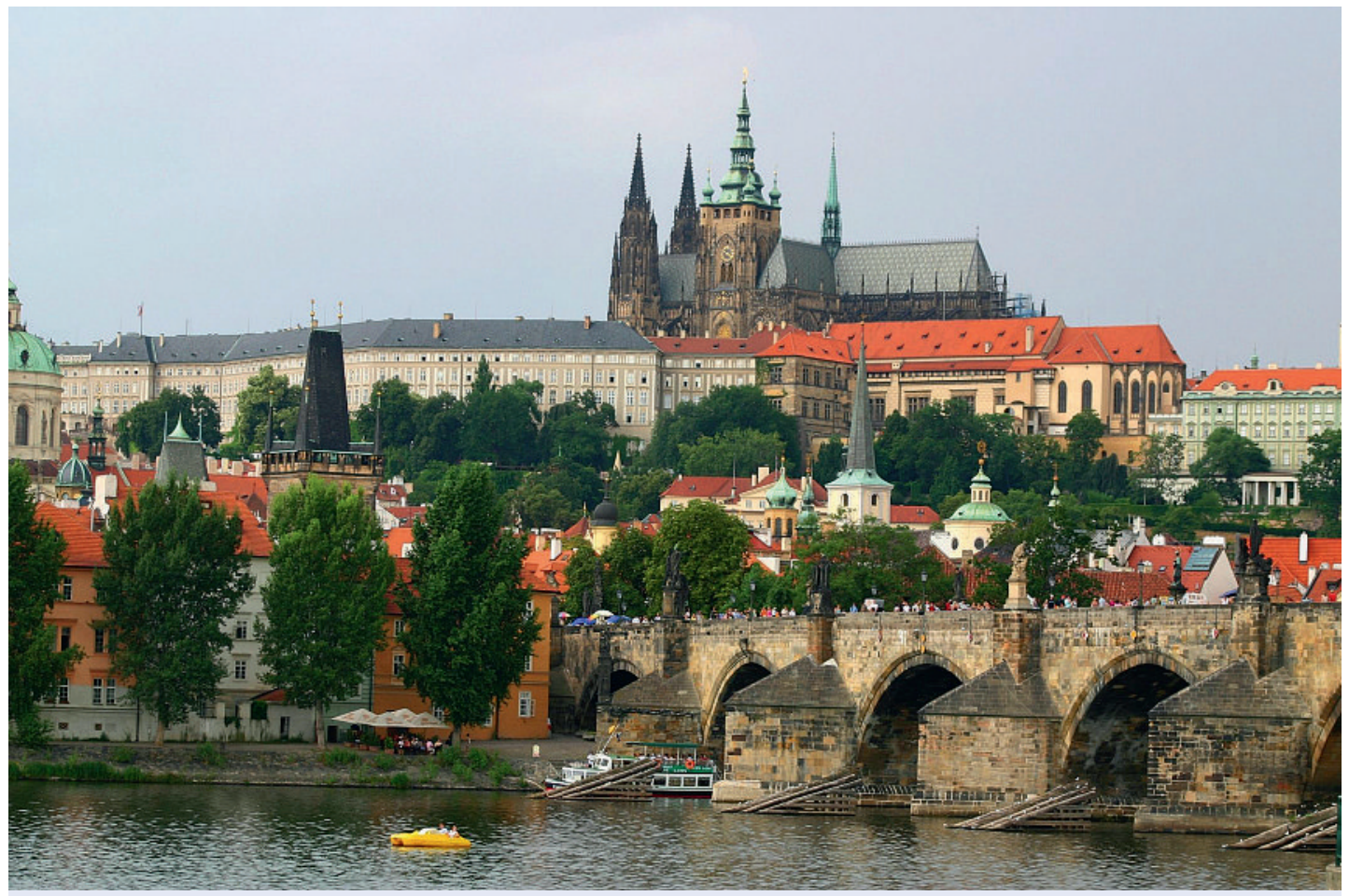

L'Union européenne des médecins spécialistes (UEMS) a invité ses membres à sa $52^{\mathrm{e}}$ assemblée générale organisée à Prague.

seule fois. Cette nouvelle réglementation sera valable dès la prochaine législature. Rien n'a changé pour l'élection des délégués nationaux: ils continueront d'être désignés par leur pays respectif et donc pour la durée de leur mandat.

\section{Ce n'est qu'en se regroupant plus étroitement et en parlant d'une seule voix que les organisations européennes de médecine pourront négocier avec succès à Bruxelles}

Afin de répondre au souhait exprimé depuis des décennies par les sociétés de discipline et leur permettre de collaborer plus étroitement au sein de l'UEMS et avec les autres organisations européennes de médecine (Union européenne des médecins omnipracticiens/UEMO, europäische Arbeitsgemeinschaft der niedergelassenen Ärzte/EANA, Fédération européenne des médecins salariés/FEMS, Association européenne des médecins hospitaliers/AEMH, Permanent working group of european junior doctors/PGW, Comité permanent des médecins européens/CPME), un immeuble sera acquis dans le quartier de l'UE à Bruxelles. En effet, ce n'est qu'en se regroupant plus étroitement et en parlant d'une seule voix que les or- ganisations européennes de médecine pourront négocier avec succès à Bruxelles. L'UEMS est disposée à défendre les intérêts des autres organisations, notamment ceux de l'UEMO et du PGW, dans le domaine de la formation et de la qualité. Le représentant de l'UEMO a fait référence à des discussions internes qui ont pour objectif lointain l'intégration de l'UEMO en tant que société de discipline médicale pour la médecine de famille dans l'UEMS.

Concernant la chirurgie de la main et la médecine d'urgence, l'UEMS a décidé de créer des diplômes européens (european boards).

La 52e assemblée générale de l'UEMS s'est ainsi focalisée, d'une part, sur des soins médicaux de haute qualité pour les patients. D'autre part, l'UEMS, en tant qu'organisation européenne de médecine la plus ancienne, renforce son engagement en faveur de la qualité la plus élevée possible de la formation médicale postgraduée et continue dans tous les pays européens. C'est la seule voie à suivre pour parvenir à la reconnaissance réciproque des diplômes de spécialiste au sein de l'Europe, dans l'intérêt des patients et sans perdre en sécurité ni en qualité.

1 Montagne S, Jucker-Kupper P, Berendonk C, Rogausch A, Beyeler C, Giger M. Trois années d'expérience avec l'Evaluation en milieu de travail (Mini-CEX et DOPS) dans la formation postgraduée médicale. Bulletin Méd Suisses. 2010;91(4):109-11. 